ISSN electrónico: 2172-9077

DOI: http://dx.doi.org/10.14201/fjc201613165185

\title{
EL RETO DE LA BRECHA DIGITAL Y LAS PERSONAS MAYORES EN EL MEDIO RURAL ESPAÑOL. EL CASO DE CASTILLA Y LEÓN
}

\section{The Challenge of the Digital Divide and Elderly in Spanish Rural Areas. The Case of Castile and León}

\author{
Dra. Noelia MORALES ROMO \\ Profesor Contratado Doctor. Universidad de Salamanca, España \\ E-mail: noemo@usal.es \\ (iD) http://orcid.org/0000-0002-0048-2607
}

Fecha de recepción del artículo: 28/07/2016

Fecha de aceptación definitiva: 26/10/2016

\begin{abstract}
RESUMEN
El presente artículo versa sobre el binomio Nuevas Tecnologías (en especial Internet) y las personas mayores que habitan en áreas rurales. A partir de una metodología tanto cualitativa (entrevistas personales y grupos de discusión) como cuantitativa (análisis de datos demográficos), se pretende profundizar en la relación entre la brecha digital y la brecha geográfica para las personas mayores residentes en el medio rural de Castilla y León ${ }^{1}$. Los resultados obtenidos muestran la existencia de elementos estructurales diferenciados en estas zonas rurales, así como otros aspectos simbólicos con frecuencia olvidados, que han de ser tenidos en cuenta a la hora de implementar proyectos de intervención. El trabajo confirma la necesidad de atender al territorio como variable, pero también a las características de unos destinatarios que tienen mayores dificultades de uso y acceso a las TIC, y avanzar así en pro de la igualdad de oportunidades.
\end{abstract}

Palabras clave: ambiente rural; personas mayores; Tecnologías de la Información y de la Comunicación; brecha digital, Internet.

\begin{abstract}
This article includes some reflections about the tandem between new technologies, particularly the Internet, and elderly people living in rural areas. This article combines a qualitative (personal interviews and focus groups) and quantitative methodology (analysis of demographic data), aiming to deep about the relationship between relationship between the digital divide and geographical gap for elderly people living in rural areas in Castile and León. Results obtained from this research show the existence of structural elements in these rural areas but also symbolic aspects often overlooked, which must take into account when implementing intervention projects. This study confirms the need to attend to the territory as a variable but also the characteristics of those who have greater difficulties in use and access to ICT, and progress towards equal opportunities.
\end{abstract}

Key words: rural areas; elderly people; ICTs; digital divide; Internet.

\footnotetext{
${ }^{1}$ Región situada en el noroeste de España que comprende un amplio territorio con numerosos municipios diseminados y con bajas densidades de población, salvo en sus capitales.
}

(C) Ediciones Universidad de Salamanca / CC BY - NC ND $\quad$ Fonseca, Journal of Communication, n. 13, 2016, pp. 165-185 


\section{INTRODUCCIÓN}

La influencia de la tecnología como uno de los impulsores del cambio social está fuera de todo cuestionamiento. Castells (2000) o Cohen (2007) son algunos de los sociólogos que la contemplan, dentro de las sociedades postindustriales, en simbiosis con la esfera social que impregna las distintas dimensiones en las que nos desenvolvemos en nuestra vida: laboral, educativa, de ocio, etc. Consecuentemente, cualquier estudio que pretenda profundizar en las Nuevas Tecnologías deberá considerar un marco contextual que vaya más allá de los dispositivos y su acceso, contemplando sus interrelaciones con la vida cotidiana y con los procesos de estratificación social (Querol, 2011). Si además se trata de conocer los vínculos con el colectivo de personas mayores, como en nuestro caso, estas premisas de partida cobran un especial protagonismo.

Diversos estudios se han afanado en estudiar la relación entre personas mayores y nuevas tecnologías en relación a los indudables beneficios que para las primeras tienen las segundas (Agudo \& Pascual, 2008; Fuente, Herrero \& Gracia, 2010; Macías \& Manresa-Yee, 2013). Otros autores se han referido a experiencias concretas (Etchemendy, 2013), o al uso de herramientas específicas como la nube (Traver, 2013).

Paralelamente, Lorenzo y Santos (2004) aseguran que el medio rural no está, a pesar de numerosas mejoras en los últimos decenios, en igualdad de condiciones en relación al número de recursos y oferta de actividades sociales, culturales o educativas.

En este artículo vamos a partir de la realidad rural como elemento que imprime una serie de significados y características, que han de ser tomadas en consideración para planificar e implementar cualquier acción de carácter socioeducativo. Tras dar a conocer algunos de los pilares fundamentales en torno a los que asienta la vida en entornos rurales españoles, nos centraremos en la realidad de la región de Castilla y León, una comunidad autónoma vasta en extensión, con gran dispersión y baja densidad poblacionales, que dificultan la provisión de todo tipo de servicios. Uno de los elementos sociodemográficos diferenciadores de esta comunidad es precisamente el envejecimiento de su población, más acuciante en los núcleos poblaciones más pequeños.

Por tanto, en este artículo nos proponemos abordar esta realidad profundizando en la idiosincrasia de este entorno rural, conociendo las relaciones entre las personas mayores y las tecnologías de la información (con especial énfasis en el acceso y uso de Internet), a través de un acercamiento a la situación cualitativa y cuantitativa de los mayores en este contexto. En definitiva, se trata de vincular entre sí tres variables, para ofrecer una panorámica que sirva para ofrecer elementos de análisis en aras a la alfabetización y comunicación digital de los mayores rurales.

Por todo ello, nuestro propósito se centra en cumplir los siguientes objetivos:

- Profundizar en la relación entre brecha digital y brecha geográfica.

- Conocer la realidad actual del medio rural de Castilla y León y, más concretamente, de sus habitantes más longevos.

- Identificar las barreras para la implantación de iniciativas vinculadas a las TIC destinadas a personas mayores.

- Sondear posibles opciones para superar la brecha digital de los mayores castellanoleoneses.

\section{HACIA UNA CARACTERIZACIÓN DE LAS RURALIDADES ESPAÑOLA Y CASTELLANOLEONESA}

El medio rural en España ha vivido profundas transformaciones en las últimas décadas. A pesar de estos cambios, los importantes desequilibrios demográficos provocados por el intenso éxodo rural de la segunda mitad del siglo pasado, determinan aún hoy las estructuras poblacionales rurales. Algu- 
nas de sus consecuencias siguen lastrando hoy la sostenibilidad social de muchas de las zonas rurales españolas: los desequilibrios demográficos, las desigualdades de género y las diferencias en cuanto a la movilidad.

De los residentes actuales en el medio rural, hay un colectivo que ha sido señalado por varios autores por su importancia estratégica. Se trata de la generación soporte (Del Barrio, 2010; Camarero, 2009). Esta generación está compuesta por los hijos de los que permanecieron en el medio rural en la época del éxodo masivo a las ciudades y cuya edad media está en torno a los 45 años. En el trabajo coordinado por Camarero (2009) sobre la realidad rural española, ocupan un lugar central, pues suponen el centro neurálgico de la vida rural por su edad activa, y por su importancia como cuidadores de mayores y menores; y lo son porque las generaciones inmediatamente superior e inferior son, en comparación, numéricamente reducidas.

La pluriactividad económica es una de las principales características de la sociedad rural actual (Moyano, 2000). La diversidad de esta sociedad, que se ha tornado mucho más compleja y se encuentra incardinada en la sociedad global, ha sido recogida por diversos autores (Cloke, Marsden \& Mooney, 2006; Woods, 2011).

De acuerdo con García Sanz (1998) podemos hablar del paso de la crisis demográfica de la sociedad rural al estancamiento y la recuperación. Algunos de sus artículos nos ofrecen gran cantidad de información sobre lo que podemos denominar la nueva ruralidad: nuevos inquilinos y retorno de antiguos emigrantes, una nueva cultura adaptada a los tiempos, que comparte un interés común por revitalizar y actualizar la identificación colectiva. Estos nuevos moradores, sin embargo, no resultan ser suficientes para producir cambios en las estructuras demográficas y poblacionales de la mayoría de zonas rurales.

Por su parte, Benito (1998) dibuja un mundo rural español que, por un lado se dirige hacia un descenso poblacional, al tiempo que emerge en otras zonas, dentro de una estructura económica globalizada por un lado y localizada por otro. Apunta este autor una serie de problemas que ha de solucionar la sociedad rural para su desarrollo: la escasez de servicios básicos o la necesidad de mejoras formativas más acordes a la realidad entre otros. El primero lo podemos vincular con el acceso a las nuevas tecnologías y el segundo con su uso.

Hay un consenso al afirmar que la realidad rural en España ha cambiado y, consecuentemente, se está extendiendo el concepto de ruralidad ampliada para hacer mención al espacio rural como un ámbito de producción y de reproducción social ya apuntado hace dos décadas por Del Barrio (1996). En otros países este concepto -que recoge la diversidad de variables y factores interrelacionados en el medio rural y que han evolucionado respecto a décadas precedentes-, también es empleado para caracterizar la complejidad de su hábitat rural. El Instituto Nacional de Estadística, Geografía e Informática de México ya lo utilizó hace dos lustros para explicar la evolución de los principales fenómenos sociales y demográficos de la población que reside en localidades rurales (INEGI, 2005). En Argentina, Craviotti (2008) lo emplea para acercarse al trabajo agrario desde un punto de vista multidimensional.

Por tanto, se hace patente la necesidad de romper con la tradicional visión de la sociedad rural y el paradigma de la Nueva Ruralidad se sitúa en esta dirección. Esta nueva ruralidad puede ser entendida como una respuesta a los preceptos neoliberales y al fenómeno de la globalización (De Grammont, 2004; Kay, 2005), o bien como una nueva propuesta de desarrollo rural estrechamente ligada a la comprensión de los actores del territorio (Arias, 2005; Pérez \& Caballero, 2003).

A su vez, el territorio rural español está conformado por espacios muy diversos en términos demográficos, ocupacionales y socioculturales. Este trabajo se desarrolla en la comunidad castellanoleo- 
nesa, caracterizada por un considerable minifundismo municipal, un elevado número de entidades de población y un índice de ruralización mucho más intenso a medida que decrece la densidad de población (Del Barrio, 2010). Algunos de los elementos definitorios de la sociedad rural española se ven pergeñados de forma más intensa en algunas autonomías como es el caso de Castilla y León. No es de extrañar, a la vista de esta consideración, que algunos estudios hayan puesto el foco en esta comunidad (García \& Lázaro, 2009; Rico González \& García, 2005; Gómez, 2011). Camarero y Sampedro (2008), por su parte, comparan la masculinización rural de Castilla y León con la existente en Valencia, por considerar ambas regiones los exponentes de mayor y menor representación del fenómeno, respectivamente. Ambos autores concluyen en una imbricación entre la masculinización y la movilidad de las mujeres, y esta, a su vez, estaría motivada por las oportunidades laborales. Se trata de una muestra más de la interrelación entre distintas variables que se anudan entre sí, dando como resultado un medio rural complejo y diverso que requiere de visiones centradas en el territorio desde una perspectiva material, pero también social.

A continuación, se analiza, de forma específica, la realidad demográfica de las personas mayores del medio rural castellanoleonés.

\section{RADIOGRAFÍA DEMOGRÁFICA DE LOS MAYORES EN EL MEDIO RURAL DE CASTILLA Y LEÓN}

Castilla y León es la comunidad más envejecida de España. Seis de las diez provincias españolas más envejecidas pertenecen a esta comunidad autónoma. Con índices demográficos preocupantes, que se analizarán en este artículo, la sociedad española de principios del siglo XXI, como sus homólogas del occidente europeo, está cada vez más envejecida. Por tanto, se trata de una tendencia muy extendida en determinadas zonas del planeta, pero con diferente alcance en territorios urbanos y rurales.

El envejecimiento del medio rural español en general y del castellanoleonés en particular, se explica por tres factores clave: la emigración del campo a la ciudad que se produce en España a partir de la década de los 50, el incremento en el último siglo de la esperanza de vida en torno a 35 años, y el descenso de la natalidad debido fundamentalmente a la planificación familiar y la incorporación masiva de la mujer al mercado de trabajo.

Tabla 1. Población en Castilla y León por grupos de edad y distribución provincial. 2015

\begin{tabular}{|l|l|l|l|l|l|l|l|l|l|l|}
\hline & Total & Ávila & Burgos & León & Palencia & Salamanca & Segovia & Soria & Valladolid & Zamora \\
\hline Edad & 2.472 .052 & 164.925 & 364.002 & 479.395 & 166.035 & 339.395 & 157.570 & 91.006 & 526.288 & 183.436 \\
\hline$<16$ & 338.563 & 21.384 & 50.892 & 55.176 & 20.007 & 42.785 & 22.576 & 11.967 & 75.429 & 18.944 \\
\hline $16-44$ & 821.265 & 55.246 & 126.281 & 159.554 & 55.176 & 113.138 & 55.819 & 30.279 & 187.934 & 57.241 \\
\hline $45-64$ & 716.006 & 46.811 & 105.481 & 141.179 & 51.012 & 97.000 & 44.510 & 25.707 & 151.611 & 52.695 \\
\hline 65 ó + & 596.218 & 41.484 & 81.348 & 123.486 & 39.840 & 86.472 & 34.665 & 23.053 & 111.314 & 54.556 \\
\hline
\end{tabular}

Fuente: INE, Padrón Municipal 2015. Elaboración propia.

De los casi dos millones y medio de habitantes en Castilla y León, un $24 \%$ son personas con más de 65 años. Si atendemos al tamaño de municipio, existe una relación directa entre el tamaño municipal y la población más envejecida, como evidencia la tabla 2. 
Tabla 2. Título. Población mayor en Castilla y León por tamaño municipal. 2015

\begin{tabular}{|l|l|l|l|}
\hline Tamaño municipal & Población total & $\mathbf{> 6 5}$ & $\mathbf{8 0 0}$ \\
\hline$<101$ & 38.795 & 15.765 & 7.053 \\
\hline $101-500$ & 259.976 & 97.000 & 42.011 \\
\hline $501-1.000$ & 160.552 & 51.514 & 21.339 \\
\hline $1.001-2.000$ & 176.966 & 47.384 & 18.759 \\
\hline $2.001-5.000$ & 217.694 & 43.896 & 16.669 \\
\hline $5.001-10.000$ & 238.504 & 40.889 & 14.581 \\
\hline $10.001-20.000$ & 119.232 & 20.555 & 7.325 \\
\hline$>20.000$ sin capital & 211.393 & 37.040 & 13.531 \\
\hline Capitales & 1.048 .940 & 235.776 & 80.201 \\
\hline Total & 2.472 .052 & 589.819 & 211.469 \\
\hline
\end{tabular}

Fuente: INE, Padrón Municipal 2015. Elaboración propia.

Un dato significativo es que, de los mayores castellanoleoneses de 65 años, el 35,9\% supera los 80 años, incluyendo las capitales. Por tamaño de municipio, en los que oscilan entre 101 y 500 habitantes, el porcentaje de mayores de 80 años con respecto a los mayores de 65 se eleva al 43,3\% y, en los menores de 101 al 44,7\%. Un último dato para constatar el peso numérico de los mayores en las comunidades más pequeñas: el 40,6\% de la población de los municipios menores de 101 habitantes tiene más de 65 años.

Las tablas 3 y 4 presentan una serie de aproximaciones cuantitativas más concretas sobre las tasas de vejez e índices de sobreenvejecimiento por provincia y tamaño municipal respectivamente. Las tasas de vejez más elevadas se concentran en Zamora, León y Salamanca, y en los municipios de menor tamaño.

Tabla 3. Tasas de vejez $z^{2}$ e índices de sobreenvejecimiento ${ }^{3}$ por provincia en Castilla y León. 2015

\begin{tabular}{|l|l|l|}
\hline & Tasa de vejez $\mathbf{~}$ & Índice de sobreenvejecimiento $\%$ \\
\hline Total CyL & 23,8 & 35,9 \\
\hline Ávila & 25,2 & 39,6 \\
\hline Burgos & 22,3 & 37,6 \\
\hline León & 25,8 & 39,7 \\
\hline Palencia & 24,0 & 39,2 \\
\hline Salamanca & 25,5 & 38,4 \\
\hline Segovia & 22,0 & 40,0 \\
\hline Soria & 25,3 & 42,6 \\
\hline Valladolid & 21,2 & 31,8 \\
\hline Zamora & 29,7 & 40,5 \\
\hline
\end{tabular}

2 Tasa de veję: Porcentaje de la población de 65 y más años sobre la población total.

3 Índice sobreenvejecimiento: Porcentaje de la población de 80 y más años sobre la población de 65 y más años. 
Tabla 4. Tasas de vejez e índices de sobreenvejecimiento por tamaño municipal en Castilla y León. 2015

\begin{tabular}{|l|l|l|}
\hline Tamaño municipal & Tasa de vejez $\%$ & Índice de sobreenvejecimiento \% \\
\hline$<101$ & 40,6 & 44,7 \\
\hline $101-500$ & 37,3 & 43,3 \\
\hline $501-1.000$ & 32,1 & 41,4 \\
\hline $1.001-2.000$ & 26,8 & 39,6 \\
\hline $2.001-5.000$ & 20,2 & 38,0 \\
\hline $5.001-10.000$ & 17,1 & 35,7 \\
\hline $10.001-20.000$ & 17,2 & 35,6 \\
\hline$>20.000$ sin capital & 17,5 & 36,5 \\
\hline Capitales & 22,5 & 34,0 \\
\hline
\end{tabular}

Fuente: INE, Padrón Municipal 2015. Elaboración propia.

Un amplio estudio sociodemográfico sobre Castilla y Léon tuvo en cuenta otra variable, la distancia a las capitales, y mostraba tasas más elevadas en municipios que distaban más de $25 \mathrm{~km}$ de las capitales (Del Barrio, 2010). Una tónica similar se observa en relación a los octogenarios.

En la mayoría de municipios de la comunidad autónoma las tasas de vejez, el índice de dependencia y el de envejecimiento, sobrepasan la media de la comunidad autónoma y de España.

La distribución de la población por edad muestra que las tendencias demográficas de Castilla y León son más pesimistas que a nivel nacional debido, sobre todo, a los menores índices de juventud, los mayores índices de dependencia, las menores tasas de reemplazo, el menor coeficiente de sustitución laboral y las tasas más elevadas de personas mayores en Castilla y León (casi seis puntos más que en España). Los indicadores que reflejan las tendencias demográficas y el envejecimiento de la población en Castilla y León son territorialmente muy desiguales. Estos procesos están influidos de manera sustancial por el tamaño de los municipios y la distancia de las distintas localidades a las capitales de provincia.

La tabla 5 evidencia cómo, a pesar de que la tasa de vejez de Castilla y Léon supera considerablemente a la española, en los últimos 10 años ha experimentado una subida menor que la nacional.

Tabla 5. Tasa de vejez en Castilla y León y España, 1960-2015

\begin{tabular}{|l|l|l|l|l|l|}
\hline & $\mathbf{1 9 6 0}$ & $\mathbf{1 9 9 1}$ & $\mathbf{2 0 0 1}$ & $\mathbf{2 0 0 5}$ & $\mathbf{2 0 1 5}$ \\
\hline Castilla y León & $8,4 \%$ & $17,7 \%$ & $22,7 \%$ & $22,6 \%$ & $23,8 \%$ \\
\hline España & $8,2 \%$ & $13,8 \%$ & $17,1 \%$ & $16,6 \%$ & $18,4 \%$ \\
\hline
\end{tabular}

Fuente: INE, Censos de 1960 y 1991 y Padrones de 2001, 2005 y 2015. Elaboración propia.

Los datos mostrados reflejan un panorama claro y rotundo sobre el envejecimiento la Castilla y León rural, y de forma más acuciante en sus municipios más pequeños.

Además de contabilizar los mayores del medio rural en la comunidad autónoma objeto de estudio, al de implementar actividades vinculadas a las nuevas tecnologías, es preciso conocer cuestiones vinculadas a sus costumbres, rutinas u orígenes, elementos que serán abordados en la aproximación cualitativa. 


\section{EL BINOMIO PERSONAS MAYORES Y TIC}

Son abundantes los estudios e investigaciones vinculados a las personas mayores que contemplan el concepto de calidad de vida como piedra angular. Siguiendo a Fernández-Ballesteros (1998) destacamos la multidimensionalidad del término y su dependencia del contexto (de ahí la importancia que en este trabajo le hemos asignado al hábitat rural) o de ciertas circunstancias del individuo. Más concretamente, varias son las condiciones que parecen favorecer una buena calidad de vida: la salud, las habilidades funcionales, las condiciones económicas, la actividad, los servicios sociales y sanitarios, la calidad en el propio domicilio y en el contexto inmediato, la satisfacción con la vida, y las oportunidades culturales y de aprendizaje. Cabe señalar que las nuevas tecnologías pueden constituir una herramienta de mejora para la mayoría de estos elementos.

El acceso a las TIC es un requisito importante para participar en una sociedad cada vez más influida por la tecnología (ALADI, 2003). En consecuencia, todos aquellos que carezcan de este acceso quedarán al margen de muchas de las posibilidades de la sociedad en que viven. Los mayores y aquellos que vivan en zonas donde no llega la banda ancha tienen mayores dificultades (Tablas 6 y 7). Estos colectivos explican el concepto de brecha digital que, como afirma el Programa para la Sociedad de la Información, se refiere no sólo a la diferencia entre individuos, sino también grupos familiares, empresas e incluso áreas geográficas, que tienen o no la oportunidad de acceder a las TIC (PSI, 2001).

Tabla 6. Uso de Internet en España en los últimos 3 meses por grupo de edad y hábitat

\begin{tabular}{|l|l|l|l|}
\hline Edad/Hábitat & $\begin{array}{l}\text { \% Personas que } \\
\text { utilizaron Internet }\end{array}$ & $\begin{array}{l}\text { \% Personas que utilizaron } \\
\text { Internet 1 vez por semana }\end{array}$ & $\begin{array}{l}\text { \% Personas que compra- } \\
\text { ron a través de Internet }\end{array}$ \\
\hline TOTAL & 80,6 & 76,5 & 34,9 \\
\hline Edad: 16 a 24 años & 98,4 & 96,8 & 44,4 \\
\hline Edad: 25 a 34 años & 96,0 & 93,8 & 49,8 \\
\hline Edad: 35 a 44 años & 93,3 & 89,2 & 46,4 \\
\hline Edad: 45 a 54 años & 84,9 & 79,1 & 34,0 \\
\hline Edad: 55 a 64 años & 64,8 & 59,1 & 19,7 \\
\hline Edad: 65 a 74 años & 34,7 & 30,7 & 7,5 \\
\hline Hábitat: > 100.000 hab. & 83,8 & 80,5 & 39,6 \\
\hline Hábitat: 50.000-100.000 hab. & 82,8 & 78,2 & 35,7 \\
\hline Hábitat: 20.000 - 50.000 hab. & 78,8 & 74,7 & 32,5 \\
\hline Hábitat: 10.000 - 20.000 hab. & 79,5 & 74,6 & 32,0 \\
\hline Hábitat: $<10.000$ hab. & 74,3 & 69,2 & 27,9 \\
\hline
\end{tabular}

Fuente: INE, Padrón Municipal 2015. Elaboración propia. 
Tabla 7. Uso de Internet en Castilla y León en los últimos 3 meses por grupo de edad y hábitat

\begin{tabular}{|l|l|l|l|}
\hline Edad/Hábitat & $\begin{array}{l}\text { \% Personas que } \\
\text { han utilizado } \\
\text { Internet }\end{array}$ & $\begin{array}{l}\text { \% Personas que } \\
\text { han utilizado } \\
\text { Internet al menos } \\
\text { una vez por sema- } \\
\text { na }\end{array}$ & $\begin{array}{l}\text { \% Personas que han } \\
\text { comprado a través } \\
\text { de Internet }\end{array}$ \\
\hline TOTAL & 77,0 & 71,5 & 30,3 \\
\hline Edad: 16 a 24 años & 100,0 & 100,0 & 48,4 \\
\hline Edad: 25 a 34 años & 94,8 & 91,4 & 54,4 \\
\hline Edad: 35 a 44 años & 92,1 & 88,5 & 41,0 \\
\hline Edad: 45 a 54 años & 85,2 & 76,8 & 27,5 \\
\hline Edad: 55 a 64 años & 59,1 & 50,1 & 11,1 \\
\hline Edad: 65 a 74 años & 33,4 & 28,2 & 7,3 \\
\hline Hábitat: $>100.000$ hab. & 100,0 & 100,0 & 48,4 \\
\hline Hábitat: $50.000-100.000$ hab. & 94,8 & 91,4 & 54,4 \\
\hline Hábitat: $20.000-50.000$ hab. & - & - & - \\
\hline Hábitat: $10.000-20.000$ hab. & 92,7 & 85,0 & 31,0 \\
\hline Hábitat: $<10.000$ hab. & 72,0 & 65,9 & 23,4 \\
\hline
\end{tabular}

Fuente: INE, Padrón Municipal 2015. Elaboración propia.

Con unos datos ligeramente inferiores en el caso de Castilla y León respecto de los datos nacionales en cuanto al uso, frecuencia y compras realizadas por Internet, las tablas 6 y 7 confirman la existencia de una brecha digital de carácter generacional además de una brecha marcada por el tamaño municipal: a mayor edad y menor tamaño municipal menor es el uso de Internet. El cambio más brusco se produce a partir de los 64 años, donde se pasa de un 59,1\% de castellanoleoneses de entre 55 a 64 años que accedieron a Internet en el último trimestre, a un 33,4\% en la franja etaria comprendida entre los 65 y 74 años.

Dentro de cada país, igual que entre los diferentes países, se perciben las desigualdades en el acceso y uso digitales como una amenaza importante (Menou, 2004). A su vez, el concepto de brecha digital se puede subdividir en tres tipos: la de acceso, basada en la diferencia entre las personas que pueden acceder o no a las TIC; la de uso, basada en las personas que saben utilizarlas o no; y la de la calidad de uso, basada en las diferencias entre los mismos usuarios (Cho, 2004). En relación a Internet, Castells (2011) la conceptualiza poniendo el énfasis en la diferencia entre los que tienen o no Internet. Por su parte, la OCDE aboga por «el desfase o división entre individuos, hogares, áreas económicas y geográficas con diferentes niveles socioeconómicos con relación tanto a sus oportunidades de acceso a las tecnologías de la información y la comunicación como al uso de Internet» (OCDE, 2011, p. 5).

Varios estudios han puesto el acento en la variable geográfica a la hora de analizar la brecha digital, refiriéndose en realidad a lo que podríamos denominar «brecha geográfica». En España encontramos una investigación significativa (Carmona \& García, 2007) que concluye en una línea divisoria entre las comunidades autónomas con un porcentaje de internautas superior a la media nacional, y aquellas (entre las que se encuentra Castilla y León) cuya media es inferior. Establece también una correlación entre mayores densidades de población y entorno más propicio para la adopción y extensión del uso de Internet.

A nivel europeo, la difusión de Internet y otros medios de comunicación es estudiada en 15 países, concluyendo una influencia significativa de factores como ingresos, nivel educativo o investiga- 
ción (Vicente \& López, 2006). Similares resultados fueron obtenidos en otro estudio sobre Europa que añade además el coste de acceso, variables demográficas y características regionales como elementos directamente relacionados con el uso y la extensión de Internet (Demoussis \& Giannakopoulos, 2006).

En Asia, Pringle \& David (2002) constatan dos experiencias de extensión de las TIC en Sri Lanka, poniendo de relieve, por un lado el potencial de Internet de ofrecer nuevas herramientas para el desarrollo rural y, por otro, los esfuerzos especiales requeridos para implantar las TIC en áreas rurales con dificultades de acceso y de toma de conciencia de su importancia.

A estos trabajos se suman los basados en la brecha digital generacional entre jóvenes y adultos (Tapscott, 2009; Busquet, Ballano, Medina \& Uribe, 2012; Boschma, 2008; Prensky, 2001, 2006; Livingstone, 2002), quienes han encontrado diferencias significativas en el uso y acceso a las nuevas tecnologías. Otras investigaciones van más allá constatando diferencias entre los propios jóvenes en cuanto a la brecha digital (Ballano, 2012).

Parece obvio pensar que las personas mayores mantendrán e incluso incrementarán esta brecha generacional con respecto a la población más joven, a la vez que aumenta su disponibilidad de tiempo y sus necesidades de comunicación se hacen más imperiosas (especialmente para los que viven en zonas rurales más lejanas, separados de su familia y con menos servicios).

Si bien se asume que el acceso a Internet reduce el sentimiento de aislamiento y fomenta la participación social, existen barreras que dificultan su uso por parte de las personas de más edad. Por una parte, las físicas en cuanto al acceso (más acusadas en los entornos rurales más pequeños y más alejados de epicentros urbanos) por la lejanía y escasez de recursos, y por otra, barreras invisibles, sociales y culturales. Para superar las segundas, señala Pavón (2000) la necesidad de motivar a los mayores a través de sus necesidades, de la vinculación a situaciones reales basadas en sus experiencias y la consideración de diferencias interindividuales. Agudo y Pascual (2008) también recogen, en aras a superar la brecha digital y más allá del acceso a infraestructuras, la necesidad de abordar las barreras de naturaleza mental a través de la educación.

Querol huye de la idea generalizada de reticencia al uso de las TIC por parte de las generaciones de más edad, y sugiere la modulación en los usos como elemento de análisis y consideración para unos grupos que no se socializaron con las Nuevas Tecnologías, pero que pueden ser objeto de lo que denomina «socialización inversa» y define como el proceso por el que «los mayores se van incorporando en el entorno familiar intergeneracional por imitación de los jóvenes» (Querol, 2011, p. 202).

White (2006) da un paso más cuando afirma que los individuos que hacen uso de Internet son incitados a interactuar, a encontrar una comunidad e identificarse con representaciones propias de este espacio. Sin duda las personas mayores, una vez superados los primeros obstáculos de uso y manejo de los dispositivos (ordenador, tablet, teléfono móvil), deberán enfrentarse a unos códigos y representaciones que les resultan lejanos y desconocidos, pero que al mismo tiempo les pueden resultar novedosos y de gran interés. Algunas investigaciones ya han constatado la necesidad y el anhelo de las personas mayores hacia el aprendizaje de las TIC y la predisposición a hacerlo en este momento de sus vidas (Agudo; Pascual \& Fombona, 2012; Colombo, Aroldi \& Carlo, 2015).

Hace varias décadas, Cochrane y Atherton (1980) ofrecieron principios para el análisis de las condiciones culturales y para la puesta en práctica de acciones para superar la pobreza en información. Entre estos se incluyen el contextualismo, la motivación y el proceso de absorción. Por su parte, Ferrés (2006) realiza una propuesta de competencias mediáticas en base a las aportaciones de 50 expertos internacionales que concreta en las siguientes: el lenguaje, la tecnología, los procesos de producción y programación, la ideología y los valores, la recepción, y la dimensión estética. 
Sobre estas bases, se realiza en España un estudio para analizar la competencia audiovisual de la dimensión de recepción que concluye con la necesidad de propuestas de formación y aprendizaje en educomunicación, debido a los bajos niveles de decodificación emocional encontrados en la población española (Marta \& Grandío, 2013). Si la población española en general tiene dificultades de decodificación, no es aventurado pensar que los españoles de las cohortes de edad más avanzadas, y aquellos que vivan en zonas con menos recursos tecnológicos (con frecuencia hábitats rurales de tamaño reducido) tendrán mayores obstáculos que superar.

Por tanto, nos encontramos en este trabajo con una aproximación a una brecha digital afectada por dos variables fundamentales: edad y entorno rural. El trabajo empírico mostrará que la relación entre estas variables está mediatizada por distintos elementos, algunos de ellos con frecuencia olvidados.

\section{MATERIAL Y MÉTODOS}

El interés de fondo de esta investigación reside en analizar las posibilidades de las nuevas tecnologías, con especial atención al acceso y uso de Internet, para las personas mayores que habitan en el medio rural castellanoleonés. La hipótesis intuitivamente más esperable es que el hábitat rural de esta comunidad, por su dispersión y bajas densidades poblacionales, es un importante limitador en la extensión de las Nuevas Tecnologías de la Comunicación para su colectivo de más edad. En otras palabras, la brecha digital es aún más difícil de superar para los mayores, si además se le une la brecha geográfica.

Para realizar esta aproximación se hace imprescindible conocer en mayor detalle las variables etaria y geográfica. En consecuencia, se ha realizado una aproximación a algunos de los elementos y variables demográficas que informan sobre la realidad cuantitativa de los mayores en esta comunidad. En base a los datos ofrecidos por el INE (Instituto Nacional de Estadística) correspondientes al Padrón Municipal del año 2012, se han calculado las tasas de vejez e índices de sobreenvejecimiento autonómicos y provinciales.

No obstante, resulta muy limitada esta aproximación cuantitativa para valorar el incremento de las posibilidades de interrelación entre los mayores y las TIC. Tras una cierta corriente cuantitativista de análisis referida a la alfabetización mediática, cada vez son más las líneas de estudio que ponen el acento en aspectos cualitativos. Para Abad (2014) el diseño de programas formativos debería partir de una aproximación de carácter cualitativo a los mayores. Además, será preciso tener en cuenta que no hablamos de un grupo homogéneo en cuanto a nivel socioeconómico, educativo, estado de salud, entorno o intereses. En esta misma línea, un estudio se refiere a la necesidad de conocer las características de los distintos grupos pues necesitan diferentes formas y niveles de apoyo para usar Internet (Eynon \& Helsper, 2010).

El trabajo que aquí se presenta pretende contribuir a expandir dicho conocimiento cualitativo y para ello se ha optado por la realización de entrevistas semiestructuradas y grupos de discusión. En función de los objetivos descritos, ambas técnicas ofrecen una fuente de significados que posibilita interpretar aspectos del objeto de estudio, no directamente observables a través de las construcciones globales de los puntos de vista de los sujetos participantes (Taylor \& Bodgan, 1986). A su vez, permiten establecer si hay grados de consenso tanto entre los profesionales que trabajan en el medio rural de Castilla y León, como en los mayores que habitan en él. Estas informaciones son fundamentales para el análisis, pues se convierten en escenarios discursivos sobre los cuales se pueden planificar las acciones de alfabetización digital (Callejo, 2002). 
Se realizaron 18 entrevistas a personas que, por sus respectivas profesiones y/o cargos, son portadoras de significados sobre el objeto de estudio. La estructura de las entrevistas giró en torno a los siguientes núcleos temáticos: caracterización del medio rural de Castilla y León, principales elementos definitorios de los mayores en el medio rural de Castilla y León, necesidades de este colectivo en diversos ámbitos, barreras para la implantación de acciones vinculadas a las TIC y posibles iniciativas a llevar a cabo para superar la brecha digital.

En total se efectuaron dieciocho entrevistas personales, dos en cada una de las nueve provincias castellanoleonesas para asegurar la representatividad provincial. Han sido nueve los perfiles de entrevistados (dos por cada perfil): agente de desarrollo local (ADL), animador sociocomunitario ${ }^{4}$, técnico de un grupo de acción local ${ }^{5}$, docente de escuela situada en el medio rural, presidente de asociación de personas mayores, alcalde, trabajador/a social, profesor/a de informática en aulas rurales y médico.

De las dos entrevistas a cada perfil, una se realizó a un profesional vinculado a municipios menores de 2.000 habitantes y la otra a municipios de entre 2.000 y 10.000 habitantes, para verificar si existen diferencias significativas en función del tamaño municipal. Todas ellas fueron grabadas y posteriormente transcritas. Se utilizaron los programas JukeBox y WaveLab para facilitar la lectura transversal de los distintos discursos, pues permiten un análisis digital paralelo de los núcleos temáticos.

Simultáneamente, se realizaron ocho grupos de discusión siguiendo -al igual que en las entrevistas personales- el criterio de ruralidad del $\mathrm{INE}^{6}$ y el nivel socioeconómico. Para su análisis se empleó el Método de Comparaciones Constantes (Strauss \& Corbin, 1998).

La tabla 8 recoge la composición de los ocho grupos focales implementados. En todos ellos se cuidó la paridad en la representación, asegurando que ningún género tuviera una representación superior al 60\%. Si bien el nivel educativo no fue una de las variables tomada para establecer la muestra (mientras que sí lo fue el socioeducativo), se evitaron asimetrías en este sentido dentro de cada uno de los grupos de discusión.

Tabla 8. Composición de los grupos de discusión

\begin{tabular}{|c|c|c|c|c|}
\hline GRUPOS & GD1 / GD5 & GD2 / GD6 & GD3 / GD7 & GD4 / GD8 \\
\hline Tamaño municipal & $<2.000$ habitantes & $2.000-10.000$ habitantes & $<2.000$ habitantes & $2.000-10.000$ habitantes \\
\hline Rango de edad & $65-83$ & $65-86$ & $66-89$ & $65-92$ \\
\hline Nivel socioeconómico & Medio-bajo & Medio-bajo & Medio-alto & Medio-alto \\
\hline
\end{tabular}

Fuente: elaboración propia.

\section{RESULTADOS}

A continuación, se muestran algunos de los principales datos cualitativos obtenidos según los cinco grandes pilares en torno a los cuales giraron las entrevistas. Esta estructuración responde a los objetivos propuestos.

\section{Caracterización del medio rural de Castilla y León}

La percepción de las áreas rurales de Castilla y León tiene un cierto tono pesimista que pivota en torno a los datos demográficos, la baja densidad de población y el déficit de recursos, evidenciado

\footnotetext{
${ }^{4}$ El Animador/a Sociocomunitario/a es un profesional presente, junto al Trabajador/a Social, en cada uno de los 192 CEAS (Centros de Acción social) existentes en Castilla y León. Se dedica a la dinamización social y cultural.

${ }^{5}$ Los Grupos de Acción Local son asociaciones sin ánimo de lucro cuyo objetivo es la aplicación de un programa regional de desarrollo rural. Gestionan fondos europeos para el desarrollo rural.

${ }^{6}$ El Instituto Nacional de Estadística español establece la siguiente clasificación según el criterio poblacional: Municipios rurales: aquellos con menos de 10.000 habitantes.

Ruralidad restringida: municipios que superar los 2.000 habitantes.
} 
tanto en las entrevistas como en los grupos de discusión. No obstante, en ambas técnicas, se establece una brecha diferencial entre las zonas y comarcas con municipios más pequeños y dispersos, y los de mayor tamaño.

La realidad es la que es. Muchos pueblos pequeños como el mío se están muriendo. Cada vez. quedamos menos y la mayoría somos muy mayores. Los jóvenes se van, las escuelas se cierran. [GD1]

Los grupos de discusión revelan diferencias significativas en la percepción del medio rural en función del tamaño municipal y de los recursos disponibles, variables frecuentemente correlacionadas. Así, se esbozan zonas rurales de tamaño intermedio que disponen de unos recursos educativos, sanitarios, de servicios sociales o culturales similares a los de las zonas urbanas, en contraste con otras áreas rurales donde estos servicios son tan inexistentes y/o deficitarios que no cubren las necesidades de su población, afectando, por consiguiente, a su calidad de vida.

No se puede hablar de ruralidad como si fuera todo igual. Hay enormes diferencias entre los modos de vida, los recursos y la realidad de los pueblos pequeños y las cabeceras comarcales. [Agente de Desarrollo Local]

\section{Características de los mayores en el medio rural de Castilla y León}

Los sujetos entrevistados ofrecen una imagen bastante homogénea de las personas mayores en Castilla y León. Los elementos más definitorios y recurrentes han sido el bajo nivel educativo, el fuerte sentimiento de vinculación al territorio, el alto índice de masculinidad, su tendencia conservadora y lo que muchos han bautizado como «carácter castellano».

El carácter castellano es un poco cerrado y muy conservador. Les cuestan los cambios, al principio son un poco reacios a todo lo nuevo, incluyendo la gente nueva que viene a vivir al pueblo. Además les cuesta unirse para buscar el bien común. [Técnico Grupo de Acción Local]

En cambio, los grupos de discusión realizados pusieron el acento en la diversidad dentro del colectivo de los mayores castellanoleoneses. Cuatro fueron los elementos más significativos de esta heterogeneidad: la edad -y en relación con ella el estado de salud-, el tamaño del lugar de residencia, el nivel educativo y la profesión que habían desempeñado.

Los que sois más jóvenes y estáis mejor de salud tenéis más ganas de aprender cosas nuevas como Internet, pero los de mi edad ya no estamos para esas cosas (...) [GD6]

A mi por ejemplo me gustaría enterarme de la lonja o del tiempo por Internet pero no me voy a comprar un ordenador a estas alturas y, además, ¿quién va a venir a mi pueblo de 150 babitantes a enseñarme? [GD1]

Yo creo que algunos de los que estamos aqui manejamos mejor un ordenador o sabemos sacarle más partido a Internet que muchos más jóvenes que viven en nuestros pueblos. No se trata solo de la edad, también de tu formación y del trabajo que hayas realizado. [GD8]

En casi todos los grupos de discusión, de forma no implícita, se identificaban barreras de carácter simbólico como elementos que alejaban las TIC de los mayores. En torno a ello pivotaron núcleos temáticos como las diferencias intergeneracionales, la idea de que las nuevas tecnologías están enfocadas a un público más joven o que utilizan un lenguaje lejano y desconocido para los mayores.

Es cierto que para nuestra generación es más difícil. Mi nieto por ejemplo es el que me ha enseñado a mí a manejar el móvil. Al principio yo pensaba que eso ya no era para mí, pero poco a poco le voy cogiendo el gustillo. Ahora dice que me va a enseñar a hablar con él por el ordenador... [GD5]

3. Principales necesidades de las personas mayores en áreas rurales 
Las entrevistas realizadas apuntan la soledad y el aislamiento como las principales necesidades de este colectivo y los grupos focales lo corroboran.

Aqui estamos olvidados, somos viejos, vivimos en pueblos de los que nadie se acuerda y si encima eres viudo como yo, pues... mal asunto. [GD1]

En muchos municipios, incluso en los de menos población, existen asociaciones de mayores que articulan la vida social de los más longevos. Se consideran como el epicentro de las actividades culturales de cada municipio y se apuntan como una buena opción para transmitir las TIC, según los profesionales entrevistados.

Desde que yo llevo aqui hemos ayudado a crear varias asociaciones de mayores. Creo que cumplen una gran labor con gente que a veces está muy sola y que no tiene mucho que hacer en estos pueblos tan pequeños, y pueden ser un buen medio para transmitir las TIC. [Animadora Comunitaria]

De nuevo se hace presente la diferencia entre municipios más pequeños y más alejados de cabeceras de comarca, y el resto del medio rural de Castilla y León.

En mi zona veo dos realidades. Los que están más cerca de (la capital) y los más grandes que tienen más parecido con la ciudad y más recursos, y los pueblos pequeños que están más lejos. [Trabajadora Social]

Parece que la variable de distancia a centros urbanos también es de gran importancia en el acceso a recursos, e incluso, como ya vimos, en cuanto al número de personas mayores. Estos aspectos ya fueron constatados por Del Barrio (2010).

Los GD1, GD3, G5 y G7, como era esperable, plantearon altos niveles de insatisfacción con respecto a los servicios de atención médica en los municipios más pequeños, mientras que en los grupos restantes (con habitantes de municipios de mayor tamaño) las cuestiones vinculadas a la salud giraron en torno a problemáticas individuales. En lo que coincidieron todos grupos fue en la necesidad de respuesta a la soledad de muchos mayores, solteros y viudos principalmente, que no tienen a su familia cerca.

Aquí los inviernos son duros. Los que estamos viudos y no nos hemos querido ir a una residencia al final nos encontramos muy solos. Tampoco bay mucho que hacer aqui. [GD3]

Resulta significativo que los componentes de los grupos ven beneficios en el uso de las TIC, sobre todo Internet, pero no las perciben como una opción que les permita paliar algunas de sus necesidades. De nuevo parece que existe una muralla simbólica que va más allá de las dificultades de acceso a la tecnología.

\section{Barreras para la implantación de iniciativas vinculadas a las TIC}

Los sujetos entrevistados también reflejan como hecho incuestionable el beneficio que para los mayores del medio rural tendría un mayor uso y acceso a las TIC. Además de las dificultades de algunos territorios, coinciden en señalar otros escollos propios del colectivo de personas mayores.

Claro que sería muy positivo que los mayores supiesen manejar los ordenadores y hablar con sus familias que están lejos, ver a sus nietos... La dificultad está en acercarles esas nuevas tecnologías de una forma sencilla, y que ellos las vean como una herramienta útil y accesible en lugar de algo que no es para su generación. [Animadora sociocomunitaria]

La percepción que tienen sobre la accesibilidad a Internet es diversa, aspecto lógico teniendo en cuenta que las entrevistas han sido realizadas en nueve provincias. Pese a la correlación que cabría esperar entre tamaño de municipio y acceso a banda ancha, los entrevistados hablan de zonas con acceso en las que hay gran diferencia poblacional entre los municipios que tienen esa cobertura. En la conectividad, más que el tamaño municipal las claves estarían (según las entrevistas) en la orografía de la zona y en la cercanía a las antenas o a poblaciones mayores con buena cobertura. 
En cambio, las infraestructuras sí suelen constituir una barrera en las poblaciones más pequeñas. Yo doy cursos pero casi siempre en los mismos pueblos y para las mismas personas. Hay personas mayores que han hecho varios cursos de nuevas tecnologias y otras que ni siquiera tienen la posibilidad. Los que viven aqui vienen dando un paseo, pero los de los pueblos de alrededor tienen que coger el coche y algunos ya no conducen, o los tienen que traer y es más difícil. [Profesor de informática]

Pero aun teniendo buena conexión e infraestructuras, existe una tercera limitación intangible, las reticencias de muchos mayores al uso de nuevas tecnologías, de nuevo la esfera simbólica.

Hay algunos que apenas pisaron la escuela. Leen y escriben regulary si les hablas de coger un ordenador dicen que eso no es para ellos. Seguro que si lo prueban a muchos les gustará pero lo difícil es engancharles para que prueben. [Presidente de Asociación de mayores]

Por tanto, cualquier iniciativa debe tener en cuenta estos condicionantes para acercar a los mayores las nuevas tecnologías de la comunicación. Aun así, la mayoría de los profesionales entrevistados considera que las TIC, en el momento actual, se pueden extender a buena parte de los mayores del medio rural de Castilla y León, pero no a todos. Esto excluye a los que tienen una salud más precaria, a los más ancianos y a los que viven en aldeas o municipios más pequeños, posiblemente los que tienen más necesidades de comunicación y relación. La brecha digital parece insalvable a corto plazo para estos subgrupos.

Los grupos de discusión revelaron dos barreras. La primera relacionada con el acceso:

A las compañias no les interesa traer Internet aqui, les sale caro para pocos vecinos. De hecho en el pueblo casi no hay cobertura para los móviles. [GD5]

La segunda, mucho más debatida y con más protagonismo, estuvo vinculada a condicionantes individuales de uso de Internet tales como el miedo, el desconocimiento, la sensación subjetiva de dificultad, la incapacidad para aprender o el índice de alfabetismo entre los mayores rurales.

Nosotros somos de otra generación. Los que babéis estudiado todavía..., pero uno como yo, cómo va a aprender ahora Internet si casi no se leer. [GD2]

Yo veo casi imposible que pueda aprender Internet. Fui pastor desde pequeño y no fui a la escuela. Casi no se leer y escribir bien, como para usar un ordenador. [GD1]

\section{Iniciativas para superar la brecha digital de los mayores castellanoleoneses}

Además de las peticiones de incremento de inversión pública, coordinación de los diferentes sectores y profesionales vinculados al mundo rural, así como la optimización de los recursos existentes (bibliotecas, telecentros o asociaciones de mayores), destacamos dos propuestas surgidas de las entrevistas.

Se podrian utilizar los ordenadores existentes en las escuelas del medio rural cuando finalicen las clases. Esto acercaría a las personas mayores que residen en municipios más pequeños la posibilidad de acceso a las TIC sin necesidad de adquirir nuevos equipamientos. [Técnico Grupo Acción Local]

Aunque apenas hay literatura sobre este tipo de experiencias, en España encontramos el trabajo de Gatt y Sordé (2012) referido a los beneficios de la participación de la comunidad en el uso de las TIC en las escuelas, que plantea diversas estrategias como la participación comunitaria en las aulas autosuficientes o la alfabetización mediática comunitaria basada en el aprendizaje dialógico.

Una posibilidad sería realizar un proyecto a nivel autonómico con la implicación de todas las diputaciones provinciales y de sus profesionales que trate de llevar los medios adecuados a los mayores que viven en zonas más aisladas y con menos recursos. [Trabajadora social]

La segunda iniciativa recuerda al programa andaluz Guadalinfo que ha creado Centros de Acceso Público a Internet en municipios menores de 20.000 habitantes (Ortega, 2004). Fuera de nuestras 
fronteras también se han implementado iniciativas similares, como los telecentros instalados por el Programa de las Naciones Unidas para el Desarrollo (PNUD) en aldeas rurales chinas destinados a los más pobres del país. Los resultados fueron positivos en términos económicos, de alfabetización digital y sociales (Soriano, 2007).

Estas propuestas se refieren a los elementos materiales de la brecha digital de los mayores rurales. Las barreras inmateriales se perciben como más espinosas. Hay un consenso en apostar por la educación como la clave, pero hay dificultades al de concretar su materialización. Un grupo de discusión planteó la perspectiva intergeneracional, señalando a los nietos y nietas como potentes atractivos de enganche que modulan hacia un uso intensivo de los mayores que desean estar conectados con familiares que viven lejos.

Si algo nos mueve a los mayores, eso son los nietos y la familia en general. Yo creo que si bubiese actividades a las que fuésemos con nuestros nietos para aprender a usar los ordenadores serian un éxito. $[\mathrm{GD} 7]^{7}$

Aunque el propósito de este artículo no es realizar sólidas propuestas de intervención, nos ha parecido interesante sondear a distintos sujetos portadores de significados en el medio rural para conocer qué alternativas sugieren. Será interesante explorar estas y otras alternativas. Una línea de investigación de cara al futuro será seguir profundizando sobre cómo implementar iniciativas de educomunicación para las personas mayores en general y para las que residen en el medio rural en particular.

\section{DisCUSIÓN DE RESULTADOS}

Ante el panorama descrito, se hace urgente una intervención multidisciplinar de los diversos sectores e instituciones que operan en el mundo rural, tanto públicos como privados, para reducir las brechas digital y geográfica mencionadas.

El número de mayores de 65 años se ha venido considerando durante años como un problema (Hoskins, 2002; Puig, 2000). El planteamiento de esta investigación es asumir el reto del envejecimiento, más acentuado en muchas de las áreas rurales, para tratar de ofrecer alternativas vinculadas a las TIC eficaces, realistas y adecuadas a la realidad en la que se pretende intervenir. Este cambio de definición de problema del envejecimiento a reto, ha sido recogido por diversos autores (Del Barrio, 2010; Tinao, 2005).

Parece comúnmente aceptado que el acceso a las nuevas tecnologías de la información y la comunicación, está estrechamente asociado con indicadores que incluyen la edad, la educación, los ingresos, y el lugar de residencia. La incidencia de estas variables sobre la brecha digital no genera el mismo consenso. El texto muestra varios trabajos que conceden una gran importancia explicativa al entorno geográfico, pero también encontramos investigaciones que analizan las diferencias entre entornos rurales y urbanos en el acceso a las TIC, y concluyen que la edad y la educación están más relacionadas con el uso de las tecnologías de la información que la localización geográfica (Hindman, 2000).

El objeto de estudio, por tanto, se revela como complejo y con cierta variabilidad en función del país, del sector poblacional y de otras variables socioeconómicas, de ahí la importancia de considerar cada zona con sus elementos definitorios. A todas ellas hay que sumarle los elementos de carácter simbólico.

En la realidad del medio rural de Castilla y León, y para sus habitantes de mayor edad, tres ideas han emergido de la investigación cualitativa. Este artículo no profundiza en ellas, pero sí las apunta como posibles vías en torno a las que puede ser interesante investigar. Para llevar a cabo estas u otras

\footnotetext{
${ }^{7}$ El resto del grupo acogió con entusiasmo la idea.
} 
iniciativas habrá que atender a las conclusiones precedentes que informan sobre otras variables menos destacadas en la literatura científica, pero de gran importancia a la hora de intervenir en alfabetización comunicativa.

Finalmente, hemos dejando patente que la igualdad de oportunidades es una panacea para el medio rural castellanoleonés y más aún para sus habitantes de más edad, que son muchos. Las Nuevas Tecnologías de la Información y la Comunicación se erigen con fuerza como herramienta para responder al reto que plantea el envejecimiento en muchas zonas rurales de España, pero hacerlo exige, además de proporcionar los medios humanos y materiales necesarios, articularlas y gestionarlas conociendo las características visibles (elementos socioestructurales del entorno) pero también las invisibles (nivel educativo, cuestiones culturales y barreras mentales) del colectivo en cuestión.

\section{ConClusiones}

Una vez examinados los datos cuantitativos y cualitativos, emergen una serie de conclusiones.

1. Los datos obtenidos de las técnicas empleadas orientan hacia una multiexclusión en el medio rural de Castilla y León para los mayores, especialmente para aquellos que residen en municipios más pequeños en cuanto al acceso a TIC, y para los de mayor edad en cuanto al uso. La relación entre el aumento de la edad y la reducción del uso de Internet ha sido constatada en otros estudios (LeraLópez, Gil \& Billón, 2009). Si cruzamos el tamaño municipal con las cohortes de edad más elevadas, nos encontramos con el grupo que tiene más dificultades de acceso y uso de TIC, tanto por elementos materiales como por factores simbólicos.

2. Si a medida que se reduce el tamaño del municipio de residencia aumenta proporcionalmente el número de personas mayores y disminuye considerablemente el acceso a Internet, tanto a través de medios públicos como privados, el resultado es claro: ante mayores necesidades de este grupo de edad, existen menores recursos y respuestas en las esferas social, económica y cultural. Por tanto, podemos inferir una «desigualdad de oportunidades» entre el medio rural y el urbano, más agravada a menor tamaño del municipio, y más aún para las personas más vulnerables, ya planteada por Morales (2007a).

3. El medio rural no puede ser tratado como un todo. Dentro de la comunidad estudiada, Castilla y León, encontramos al menos dos realidades rurales diferenciadas en los datos cualitativos y cuantitativos presentados. Una se corresponde con las localidades menos pobladas, más envejecidas y con frecuencia alejadas de municipios mayores que son los que concentran los recursos. La otra realidad rural es la de municipios intermedios cuyos recursos, datos demográficos y condiciones de vida se asemejan más a los de los centros urbanos. Es en estas segundas áreas municipales donde, a tenor de los datos obtenidos, será más fácil acercar a los mayores las nuevas tecnologías para incrementar sus posibilidades de comunicación y relación. No obstante, tampoco podemos olvidar a las zonas más puramente rurales, pues son las que concentran mayor número de personas mayores que se sienten más aisladas y cuentan con menores opciones de acceso a recursos que mejoren sus posibilidades de comunicación, siendo las más vulnerables a la sociedad de la información.

4. Si bien las variables edad y entorno rural parecen explicar buena parte de las necesidades de los mayores castellanoleoneses, ambas han sido subdivididas -en las técnicas realizadas- en dos escenarios. En primer término, los «mayores más jóvenes» y con mejor salud, y los que viven en zonas rurales con más recursos de acceso a TIC (las de mayor tamaño y/o las más cercanas a zonas urbanas). El segundo escenario estaría compuesto por los mayores más longevos, con más limitaciones de salud y residentes en municipios más pequeños y con menos recursos. El primer grupo estaría mejor posicionado que el segundo para el acceso y uso de nuevas tecnologías y, por tanto, tendría más fácil romper las brechas digital y geográfica. Paralelamente, el nivel educativo actuaría como freno o como acelera- 
dor en los procesos de acercamiento a las nuevas tecnologías para ambos grupos, según las consideraciones de los expertos. En consecuencia, las personas mayores constituyen un colectivo con gran heterogeneidad, tal y como han mostrado otros estudios (Morales, 2011).

5. Para responder a algunas de las carencias del colectivo estudiado, hay consenso a la hora de erigir a las nuevas tecnologías como un mecanismo relativamente económico, factible y con numerosas posibilidades para mejorar su calidad de vida. En cambio, si bien valoran positivamente las posibilidades de las TIC en general y de Internet en particular, los mayores castellanoleoneses que han participado en el estudio no los perciben como una necesidad para ellos. Un posible acercamiento, dadas las características actuales del medio rural español y castellanoleonés, podría venir de implicar a la generación soporte en el proceso de enseñanza-aprendizaje de sus padres, madres, suegros, suegras y vecinos en general. Tal y como indicamos al inicio de este artículo, y en la línea de lo apuntado por Camarero (2009), esta generación constituye un sector estratégico, que se podría convertir en la piedra angular para el acercamiento de las TIC a los mayores del medio rural, eliminando algunas de las barreras que han sido descritas. Una de las propuestas, en esta misma dirección, apunta a los nietos como enlace entre los mayores y las TIC.

6. El estudio ha reflejado algunas dificultades estructurales, demográficas, geográficas y simbólicas para la alfabetización digital de los mayores rurales. Las últimas, a veces son infravaloradas. Nuestro trabajo ha suscitado, por ejemplo, la comprensión de la impronta del «carácter castellano» para el colectivo objeto de estudio. La existencia de este imaginario social ha sido constatada por López (2014) y su articulación como barrera para el desarrollo de nuevas iniciativas por Morales (2007b). En esta misma línea discursiva, un análisis basado en áreas rurales de Centroamérica sostiene que las TIC deben proporcionar herramientas que mejoren la calidad de vida y que reivindiquen la cultura autóctona basadas en las características de la comunidad (Carvajal, 2009).

Con estos argumentos precedentes, este trabajo orienta hacia un sumatorio poco alentador de la brecha digital de las personas mayores y la brecha geográfica en el territorio estudiado. Las iniciativas basadas en las TIC como forma de e-inclusión en la sociedad de conocimiento, tendrán que tomar en consideración una serie de variables multidimensionales, que van desde aspectos materiales (ligados al territorio y a la variable etaria) a otros simbólicos que no han de ser desdeñados.

\section{REFERENCIAS BIBLIOGRÁFICAS}

Abad, L. (2014). Diseño de programas de e-inclusión para alfabetización mediática de personas mayores. Comunicar, 42, 173-180. doi: https://doi.org/10.3916/C42-2014-17

Agudo, S., \& Pascual, M. A. (2008). Posibilidades formativas de las tecnologías de la Información y comunicación en las personas Mayores. Revista de Medios y Educación, 33, 111-118.

Agudo-Prado, S., Pascual-Sevillana, M. D., \& Fombona-Cadavieco, J. (2012). Usos de las herramientas digitales entre las personas mayores. Comunicar: Revista Cientifica de Comunicación y Educación, 20(39), 193-201. doi: https://doi.org/10.3916/C39-2012-03-10

ALADI. (2003). La Brecha Digital y sus Repercusiones en los Países Miembros de la Asociación Latinoamericana de Integración. Brasil: ALADI.

Arias, P. (2005). Nueva ruralidad: antropólogos y geógrafos frente al campo. En H. Ávila (Coord.), Lo urbano-rural, ¿nuevas expresiones territoriales? (pp. 73-118). México D. F.: UNAM-CRIM. 
Ballano, S. (2014). Young users and the digital divide: readers, participants or creators on Internet? Communication \& Society, 27(4), 147-155. doi: https://doi.org/10.15581/003.27.4.147-155

Benito, J. (1998). Tendencias del mercado de trabajo en el medio rural de Castilla y León. Aspectos sociológicos. Valladolid: Consejería de Economía y Hacienda de la Junta de Castilla y León.

Boschma, J. (2008). Generación Einstein. Más listos, más rápidos y más sociables. Comunicar con los jóvenes del siglo XX. Barcelona: Ediciones Gestión 2000.

Busquet, J., Ballano, S., Medina, A., \& Uribe, A. C. (2012). La dinámica de la Brecha digital entre Jóvenes, Padres y Profesores en España. En García, A. (Ed.), Comunicación, Infancia y Juventud. Situación e investigación en España (pp. 39-55). Barcelona: UOC.

Callejo, J. (2002). Observación, entrevista y grupo de discusión: el silencio de tres prácticas de investigación. Revista Española de Salud Pública, 76, 409-422.

Camarero, L. (Coord.). (2009). La población rural de España. De los desequilibrios a la sostenibilidad social. Barcelona: Fundación la Caixa.

Camarero, L., \& Sampedro, R. (2008). ¿Por qué se van las mujeres? El continuum de movilidad como hipótesis explicativa de la masculinización rural. Revista Española de Investigaciones Sociológicas (REIS), 124(1), 73-105.

Carmona, M., \& García, L. (2007). Difusión del uso de Internet en España: ¿Existe una brecha digital entre Comunidades Autónomas? Revista de Estudios Regionales, 80, 193-228.

Carvajal, V. (2009). Inclusión o exclusión social: el reto de las TIC y el caso de las poblaciones rurales centroamericanas. Hekademus: Revista Cientifica de la Fundación Iberoamericana para la Excelencia Educativa, 5, 44-64.

Castells, M. (2000). Materials for an Exploratory Theory of the Network Society. The British Journal of Sociology, 51(1), 5-24.

Castells, M. (2011). La Galaxia Internet. Barcelona: Plaza y Janés.

Colombo, F., Aroldi, P. \& Carlo, S. (2015). Nuevos mayores, viejas brechas: TIC, desigualdad y bienestar en la tercera edad en Italia. Revista Comunicar, 45(23), 47-55. doi: https://doi.org/10.3916/C45-2015-05

Cho, C. (2004). How to measure the digital divide? Korea: Agency for Digital Opportunity \& Promotion.

Cloke, P., Marsden, T., \& Mooney, P. (Eds.). (2006). Handbook of rural studies. London: Sage.

Cochrane, G., \& Atherton, P. (1980). The cultural appraisal of efforts to alleviate information inequity. Journal of the American Society for Information Science, 31, 282-92.

Cohen, D. (2007). Tres lecciones sobre la sociedad postindustrial. Buenos Aires: Katz.

Craviotti, C. (2008). Empleo agrario y ruralidad ampliada. Geografiando: Revista de estudios geográficos, 4(4), 99-116.

De Grammont, H. (2004). La nueva ruralidad en América Latina. Revista Mexicana de Sociología, 66, 289300. doi: https://doi.org/10.2307/3541454 
Del Barrio, J. M. (1996). Espacio y estructura social. Análisis y reflexión para la acción social y el desarrollo comunitario. Salamanca: Amarú.

Del Barrio, J. M. (2010). La población de Castilla y Léon en los inicios del Siglo XXI. Valladolid: Fundación Perspectivas.

Demoussis, M., \& Giannakopoulos, N. (2006). Facets of the Digital Divide in Europe: Determination and Extend of Internet Use. Economics of Innovation and New Technology, 15(3), 235-246.

Etchemendy, E., Castilla, D., Baños, R. M., \& Botella, C. (2013). Sistema Mayordomo: la puerta de entrada de nuestros mayores a las nuevas tecnologías. Ariadna, 1, 33-38. doi: http://dx.doi.org/10.6035/Ariadna.2013.1.6

Eynon, R., \& Helsper, E. (2011). Adults Learning Online: Digital Choice and/or Digital Exclusion? New Media \& Society, 13, 534-551. doi: https://doi.org/10.1177/1461444810374789

Fernández-Ballesteros, R. (1998). Vejez con éxito o vejez competente: un reto para todos. Envejecimiento y prevención. Barcelona: Asociación Multidisciplinaria de Gerontología.

Ferrés, J. (2006). La competencia en comunicación audiovisual: propuesta articulada de dimensiones e indicadores. Quaderns del CAC, 25, 9-17.

Fuente, A., Herrero, J., \& Gracia, E. (2010). Internet y apoyo social: sociabilidad online y ajuste psicosocial en la sociedad de la información. Acción psicológica, 7(1), 9-15.

García Sanz, B. (1998). La Sociedad Rural de Castilla y León ante el próximo siglo. Valladolid: Junta de Castilla y León, Consejería de Agricultura y Ganadería.

García, R. H., \& Lázaro, J. M. (2009). El nivel de vida en el medio rural de Castilla y León: una constatación antropométrica, 1840-1970. Historia agraria: Revista de agricultura e historia rural, 47, 143-166.

Gatt, S., \& Sordé, T. (2012). ICT alone is not enough, the whole village is needed: a community-based and dialogic approach to technology in schools. International educational Psychology, 2, 153-174.

Gómez, P. G. (2011). Las políticas territoriales de desarrollo rural de la Unión Europea: un balance de veinte años en Castilla y León. Estudios de economía aplicada, 29(1), 1-23.

González, M. R., \& García, J. M. (2005). La mujer en el medio rural de Castilla y León: diversificación sectorial y proceso de dinamización económica. Estudios de economía aplicada, 23(2), 465-490.

Hindman, D. (2000). The rural-urban digital divide. Journalism and Mass Communication Quarterly, 77(3), 549-560.

Hoskins, D. (2002). Reflexiones acerca de los problemas del envejecimiento. Revista internacional de Seguridad Social, 55, 13-22.

INEGI. (2005). Población rural y ruralidad ampliada en México. Aguascalientes: INEGI.

Kay, C. (2009). Estudios rurales en América Latina en el periodo de globalización neoliberal: ¿una nueva ruralidad? Revista Mexicana de Sociología 71, 607-645. doi: https://doi.org/10.5354/03657779.2012.21734

Lera-López, F., Gil, M., \& Billón, M. (2009). El uso de Internet en España: Influencia de factores regionales y sociodemográficos. Estudios Regionales, 16, 93-115. 
Livingstone, S. (2002). Young people and new media. Childhood and the changing media environment. London: SAGE Publications.

López, M. A. (2014). La comarca como entidad natural, histórica y administrativa. Madrid: Dykinson, SL.

Lorenzo, M., \& Santos, M.A. (2004). Buscando la mejora de la escuela rural a través de los nuevos entornos educativos. Revista de Educación, 335, 215-228.

Macías, L., \& Manresa-Yee, C. (2013) Mayores y nuevas tecnologías: motivaciones y dificultades. Ariadna 1, 6-11. doi: http://dx.doi.org/10.6035/Ariadna.2013.1.2

Marta, C., \& Grandío, M. M. (2013). Análisis de la competencia audiovisual de la ciudadanía española en la dimensión de recepción y audiencia. Communication \& Society, 26(2), 114-130.

Menou, M. (2004). La alfabetización informacional dentro de las políticas nacionales sobre tecnologías de la información y comunicación (TIC): la cultura de la información, una dimensión ausente. Anales de Documentación, 7, 241-261.

Morales, N. (2007a). Escuela, medio rural e igualdad de oportunidades: ¿un trío imposible? Documentación social, 146, 135-154.

Morales, N. (2007b). La idiosincrasia de los servicios sociales en el medio rural salmantino. Cuadernos de Trabajo Social, 20, 35-56

Morales, N. (2011). Significados de jubilación y expectativas de futuro. Documentación Social, 160, 209231.

Moyano, E. (2000). Procesos de cambio en la sociedad rural española. Papers: Revista de Sociología, 61, 191-220.

OCDE (2011). Understanding the Digital Divide. Retrieved from http://www.oecd.org/dataoecd/38/57/1888451.pdf

Ortega, J. A. (2004). Nuevas tecnologías y desarrollo rural: la iniciativa andaluza Guadalinfo. En J. Cardona Andújar (Dir.), La calidad territorial como estrategia: la formación clave en el desarrollo (pp. 191193). Talavera de la Reina: Centro Asociado de la UNED.

Pavón, F., \& Castellanos, A. (2000). El Aprendizaje de los Mayores y las Nuevas Tecnologías. En E. Valenzuela \& E. Alcala (Eds.), El Aprendizaje de las Personas Mayores ante los retos del nuevo milenio (pp. 197-236). Madrid: Dykinson.

Pérez, A., \& Caballero, J. M. (2003). La Nueva Ruralidad en Europa y su Interés para América Latina. Roma: Unidad Regional de Desarrollo Agrícola y Rural Sostenible - Banco Mundial y Dirección del Centro de Inversiones - FAO.

Prensky, M. (2001). Digital Natives, Digital Immigrants. On the Horizon, 5, 1-6.

Prensky, M. (2006). Don't bother me mum, I'm learning!: how computer and video games are preparing for 21st century success and how you can help! St. Paul: Paragon House.

Pringle, I., \& David, M. J. (2002). Rural Community ICT Applications: The Kothmale Model. The Electronic Journal on Information Systems in Developing Countries, 8(4), 1-14. 
PSI. (2001). Estado inicial, informe de avance, formulación estratégica y plan de acción. Buenos Aires: Programa Nacional para la Sociedad de la Información de la República Argentina.

Puig, J. M. (2000). El envejecimiento poblacional como problema sanitario. Medicina integral: Medicina preventiva y asistencial en atención primaria de la salud, 36(5), 190-198.

Querol, V. A. (2011). Las generaciones que llegaron tarde. Análisis de las prácticas sociales de los mayores en el ciberespacio. Barcelona: Editorial UOC.

Soriano, C. (2007). Exploring the ICT and Rural Poverty Reduction Link: Community Telecenters and Rural Livelihoods in Wu'an, China. The Electronic Journal on Information Systems in Developing Countries, 32(1), 1-15.

Strauss, A. \& Corbin, J. (1998). Basics of qualitative research. Techniques and procedures for developing Grounded Theory. London: Sage.

Tapscott, D. (2009). Grown Up Digital: How the Net Generation is Changing Your World. New York: McGraw-Hill.

Taylor, S. \& Bogdan, R. (1986). Introducción a los métodos cualitativos de investigación. Barcelona: Paidós.

Tinao, J. F. (2005). El envejecimiento de la población: de los problemas a las oportunidades. Revista de bistoria actual, 3, 127-143.

Traver, J. (2013). Los mayores en la nube. Ariadna, 1, 98-105. doi: http://dx.doi.org/10.6035/Ariadna.2013.1.17

Vicente, M. R., \& López, A. J. (2006). Patterns of ICT Diffusion across the European Union. Economics Letter, 93, 45-51.

White, M. (2006). The body and the screen: theories of Internet spectatorship. Cambridge, EUA: MIT Press.

Woods, M. (2011). Rural. Abingdon: Routledge.

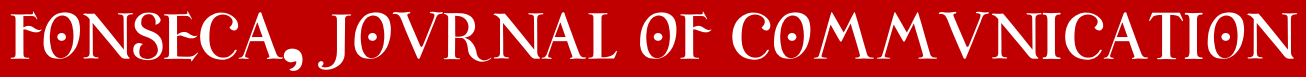

